Emergent Aesthetics: Aesthetic Issues in Computer Arts

Author(s): Mihai Nadin

Source: Leonardo. Supplemental Issue, Vol. 2, Computer Art in Context: SIGGRAPH '89 Art Show Catalog (1989), pp. 43-48

Published by: The MIT Press

Stable URL: https://www.jstor.org/stable/1557943

Accessed: 11-11-2019 22:37 UTC

JSTOR is a not-for-profit service that helps scholars, researchers, and students discover, use, and build upon a wide range of content in a trusted digital archive. We use information technology and tools to increase productivity and facilitate new forms of scholarship. For more information about JSTOR, please contact support@jstor.org.

Your use of the JSTOR archive indicates your acceptance of the Terms \& Conditions of Use, available at https://about.jstor.org/terms

The MIT Press is collaborating with JSTOR to digitize, preserve and extend access to Leonardo. Supplemental Issue 


\title{
Emergent Aesthetics- Aesthetic Issues in Computer Arts
}

\author{
Mihai Nadin
}

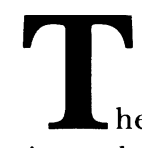
production, takes place in the context of human interaction-with others, with nature, with tools, with artifacts, and with ideas from times passed. Artistic work, more than any other, is probably a projection of the experiential structure of the act of producing artifacts (or events) with qualities socially acknowledged as artistic and values culturally celebrated as aesthetic. Throughout history, the patterns of human interaction have continuously changed, and so has art. Nonetheless, changes like the ones we experience today are unprecedented, requiring that we understand that the condition of art is probably more dependent than ever on the condition of humanity in general, and of science and technology in particular.

The age of information processing implies networking and interactivity. In a broad sense, this age can be understood as one of a generalized electronic medium against whose background digital and non-digital activities take place. It is not that, in the age of information processing, tradition or tradition-rooted forms of human practice cease; they are complemented by new forms, some impractical or even impossible in previous paradigms of thinking and creating. Two lines-one of continuity that establishes itself as an implicit reference and another of uncompromising revolution/radical change-could represent the topology of the space of artistic or scientific exploration as it results from the integration of the information-processing paradigm and the computer associated with it in our culture. These two lines follow various directions, which sometimes meet, run in parallel directions, and at some time diverge. I am suggesting this visual representation to make clear from the outset that the process is not of exclusion, but of diversification.

This said, it is time to examine what we address as computer art and to try to understand why, despite expectations (some very high) and tedious work, despite major investment (easily approaching the billion dollar mark and exceeding any other investment made in art), and despite enthusiasm, the results have been rather minor. This judgment can be questioned and contradicted, unless and until the perspective from which it is justified is defined. Indeed, if we include in our notion of computer art computer graphics in general, modeling, desktop publishing, simulation, image processing, and animation, as well as sound and image synthesis (I have not mentioned everything that might qualify), the argument of economic success, novelty and cultural impact will be impossible to refute. Moreover, the invisible participation of the computer in photography, film, video, music and graphic design technologies will definitely challenge the notion that the results achieved are minor. This is where the two lines of development-tradition and renewal-meet. New technologies are integrated into established forms of artistic practice and make possible a rationalization of previous work and a wider dissemination through channels of mass communication. The photographic camera controlled by a chip achieves what Eastman made the program of his house. Computer-supported graphic design, especially typesetting, has introduced means of increased productivity, quality control and variation unknown before. Nevertheless, once these and other examples are acknowledged, a feeling of dissatisfaction lingers. Computer-generated art and electronic music are interesting, and some works are provocative in their novelty. But once we have seen a computer graphic image or listened to a computer-generated piece of music, it seems that we have seen and heard them all. In animation, after an initial period of surprise and hope, we now know that not much progress has been made from the first flying logos to the most recent (and ridiculous) flying flame of the NBCtelevised Olympics, although technology has matured quite a bit and we have accumulated more than a fair share of experience. As opposed to works of art that look better the more we look at them, electronic art seems to exhaust itself at the first encounter.

These critical remarks describing the current state of computer art would not be more than an expression of disappointment and even subjective evaluation were it not for the need they trigger to go beyond these weaknesses and to approach basic issues as they pertain to the new aesthetic experience with the computer. These include the following:

1. the relation between a traditional notion of art and the emergent aesthetics of new forms of artistic practice

2. the relation between explanatory models of art and the generative power of explanations

3. the relation between technology and art, with special emphasis on digital technology

4. the relevance of an aesthetic consciousness for diversified artistic practice

In approaching these questions, and keeping in mind their implications and ramifications, I am aware that no simple answers can be given; furthermore, while any discourse about individual works of art can take place only after the work, nothing precludes a discussion of art as a form of participation in the process through which the artwork becomes possible. My own involvement with art and computers extends over 20 years. Although the fact that I have written programs and produced images or musical pieces does not necessarily make me the bearer of truth, my experience reflects an understanding of the subject and guarantees that my views will be accessible even to those who disagree with

Mihai Nadin (educator, consultant), Fashion Institute of Technology, School of Art and Design, 7 th Avenue at 27 th Street, New York, NY 10001-5992, U.S.A. 
them, because we share in the language of the technology and in the commitment to research its potential.

\section{ConTEXT OF CONFLICT}

It is a commonplace that new forms of art emerge in a context of conflict with established art. As with many other patterns of human interaction, artistic activity is prone to establish its own power base and to exercise it economically, politically, ethically and in other ways. This happens through institutions and through reified moral values as reflected through laws, religions, schools and universities. Computer art is probably the best example of the attempt made by established art to appropriate and limit the efficiency of the new technology. In actuality it is the fight between that which is old, respectable, valuable, significant, progressively integrated into culture and tradition, and the new promise, challenge, and hope-the beginning of a new civilization. I certainly doubt that the plethora of mediocre images in continuation of the traditional realistic, surrealistic, expressionistic, etc. art can be attributed to imitation as an obligatory phase in every new development. It is more than an imitation phase-which we all resignedly accept as a given (children imitate adults, don't they?) —and different from mimesis. My thesis is that, in the process of appropriating this particular new technology-which is fundamentally changing the nature of human praxis-traditional artists, technologists and scientists have acted to preserve modes of expression they believe in, like or have tried to explain. Patterns of human interaction, in particular those pertinent to work, social existence, artistic activity and communication, are so deeply ingrained that uncertainty about and unpreparedness for the new explain the opposition to everything that does not preserve prior experiences wholly or at least in part. Symptomatically, we have tried to convert the revolution into an evolution, to see it as a cycle in a dynamics of progress, not as a dislocation of rigid and exhausted forms of thinking, working or creating. In the arts, probably more than in any other field of human interaction, one notices how encompassing the change can be. While traditionally open to experiment, renewal and innovation, and often assuming social roles of exemplary activism, artists did not oppose the technology, but hoped that it would not affect their studios and ways of working; some even hoped that it would go away. Since this has not happened, those who wanted to give it a chance have discovered that the issue is one of change-in the technology or in themselves. Since the latter requires more than good will and investment into what we know today as user-friendliness, they opted for the former. The programs we use-rendering, image mapping, ray tracing, to name a few-do not contradict previous modes of expression but actually capture them in some computational form and make them available in everfriendlier forms to 'Sunday painters' (as Negroponte once aptly called them [1]). We simply took the new tool and forced it to solve old problems, whose answers we knew ahead of time. With each work produced with the help of the machine that matched the answer, we became more emphatic.

Obviously, at the beginning, power relations specific to artistic praxis were exercised gently. The maturing of the technology and its gradually higher price-I refer not to components, which became cheaper, but to the more complex configurations required by the complexity of the task, which require considerable investment-give these power relations aggressive, even brutal aspects. Although the new paradigm refers to and applies a reality different from the physical matter involved in previous forms of artistic practice (i.e. clay, cameras, marble, pigment-based colors, etc.), we stubbornly try to rediscover the old (assumed to be not only good, but also universal and eternal) and to preserve it. It should not be a surprise that fraud and ignorance have often played important roles in this practice of preservation. In order to make the new available, we have enrolled everyone willing to support it. The result is not unexpected: almost without exception, computer art classes are taught by those who never succeeded in their art. The new talent exhibited by technologists, scientists and self-made artists is met with suspicion and typically 'brought into line'. Even the new possibilities opened up by technology have been reduced to acknowledged procedures. Visualization of highly diversified spaces, 3D (virtual space) explorations and color explorations are still treated according to the aesthetics of white paper or canvas. This is why, although formally correct, some of the new imagery is expressively inadequate. Technical ignorance and aesthetic limitations explain the success of paint box programs, drawing programs and illustration software. Through such programs, previous forms of artistic practice are maintained, though at a qualitative level far below that of traditional tools and media. Thus, while trying to preserve a familiar mode, we in fact have preserved only the appearance of the previous mode, since the machine was no longer being used ("I will do what you want me to do") but started using the user ("Do what I can do, and how I do it")-the artist in particular. We knew that the hardware was not conceived for such creative work, but we hoped that some programs would do the trick. To a certain extent, this has been accomplished. But if art, at least in the romantic sense we still cling to, is the expression of personality, emotion, experience and the like, then the computer does not necessarily help the artist to bring it about more freely. Quite often, what is produced on the computer can be generated more easily, quickly and cheaply with a pencil or other traditional means. There are numerous instances in which the computer controls the artist and 'signs' the work. This is what I have calledand my formula has been widely adopted-'canned art'. There are also instances where the machine offers a fast substitute for art. This is what I called 'MacDonald art' [2].

\section{IS THE ARTIST A USER?}

Obviously a paint program, a drawing program, an image renderer, a ray tracer, etc. are computational models that capture knowledge about how aspects of images generated in the past can be replicated. Whereas the artist working in traditional media invented new forms of expression, the computer program gives a prefabricated, general solution. Such programs are the how of art and as such are quite impressive in their performance. As explanatory models, they rely on physics (the laws of reflection, refraction, etc.), linear mathematics (linear perspective, sectioning, solid modelling) and logic (mainly Boolean operations). To what extent a good 
explanatory model is also a generative procedure is a question raised again and again during the history of art and in epistemology. As it turns out, each explanation is incomplete. The perspective from which the explanation is given defines the level of incompleteness. Within the perspective assumed, a good description can become a generative scheme [3]. The golden section, the Fibonacci series, the formalism of the metaphor (logical or mathematical) can be used to generate artifacts with expected or desired formal qualities. Based on this limited notion of generativity, various descriptions were used at the outset of computer art and experiments were performed in order to generate families of images or sound sequences. The socalled intuitive element, i.e. variations within a given frame, was ensured (rudimentarily I should add) by the use of random number generators. What resulted was a whole family (infinite, in principle, or with a degree of infinity comparable to the degree of randomness achieved) from which an 'artist' selected what seemed aesthetically relevant. My own evolution went through this stage [4], which I actually enjoyed because of the unexpectedness that randomness sometimes led to. It was a form of aesthetic lottery whose winners attracted public attention, although it was not certain what was won. Nevertheless, the major question of whether a person (or machine) who describes art also creates art continued to obsess us. It seems that throughout the history of art the act of doing and the act of contemplating (i.e. what is known as theory) have been complementary rather than equivalent, and the hope of breaking this pattern enthused many.

Things became more complicated once the instrument of explanation and the instrument of production became the same. Indeed, the same mechanism can be used to analyze and to synthesize. Data resulting from analysis (output from a process) can become the 'matter' of the act of obtaining new artifacts (input of a reverse process). However, the logical laws governing the function of the machine require observation of stringent conditions for computability. Completeness and consistency, to which a fundamental logical law applies (Gödel), are not, by any stretch of our willingness to acknowledge logic and rationality in art, characteristics of the art process. Thus, the machine is intrinsically adapted to a universe of experience in which only partial artistic practice is possible. Indeed, the very structured nature of the typographic art makes it a good target for computer-based praxis-a theoretic idea that I expressed long before desktop publishing was made into an available key-system technology [5].

Since any description-in the philosophic form of discourse or in mathematical-logical formalism-is, after all, incomplete and thus subjective, once such descriptions become generative tools in the form of procedures or programs, they act upon the data (the 'matter' of electronic art) as a mold. The fingerprint of those who designed them gets marked in the image or the sounds generated. The 'artist', consequently, is actually the machine, while the human being becomes the operator working for the programmer(s). The uniformity of images and musical compositions that strikes anyone who has gone beyond the initial moment of surprise and even exaltation is the result of the limited number of programs and procedures available. Such programs, while deserving in many respects, were transported from machine to machine (sometimes losing efficacy compared to their initial characteristics, other times being improved) and became available in the broader market. There is no difference between a ray tracer in a sophisticated research or computer art center and the ray tracer we can buy for our PCs.

Being such high performance machines, computers are used to generate incredible numbers of images produced in all kinds of environments. However, we must make some distinctions. We look at images on screens in various contexts. Each context has its own requirements. Computer-aided design (CAD) images are, in virtue of their goal, supposed to be exact. Tools for achieving precision are continuously created. From CAD, we move towards computer-aided manufacturing (CAM), which implies precision as well but also some other characteristics, such as versatility for driving complex machines, precise time sequencing, even parallelism. Communication and entertainment applications (such as advertisement, mass communication, show business) have still different requirements, including realism. Simulation, by its very nature, suggests the need for convention based on mapping procedures from the realm of simulated phenomena to the realm of knowledge. Dynamics, as characteristic of simulated complex phenomena, requires integration of movement. In addition, the code of simulation (as it applies to colors, visual rhythms, shapes, topological changes) has to be conveyed together with the simulation, which raises issues of communication. In each of the fields mentioned, software tools (indeed, the computer is not a toolonly programs qualify as tools) were built, tested and improved according to the specific requirements of the work.

Artistic images are defined by their aesthetics; and the need for aesthetic characteristics is acknowledged. Yet while the aesthetic component is a structuring component that facilitates better usage, it is in fact only partially pursued. What those interested in the art did not understand about the tools was that it is not the precision of CAD that will make for art, not the sophistication of an integrated processing package that will make art programs out of CAM programs (even when used to drive a milling machine in order to create a 'sculpture'), nor the enticing commercial 'art packages'. Because the explanations used were explanations pertinent to any other class of artifacts but the artistic class, the result was contorted images, very technical, precise but not expressive, flashy but not convincing. In these images, a world of plastic, metal, even of gelatin [6] was constituted because $\mathrm{CAD}, \mathrm{CAM}$ and simulation programs required the texture mapping of plastic, metal and other materials that our factories process, not because it was aesthetically relevant. Instead of allowing us to see whether explanations of works of art can become generative procedures, those who use explanations pertinent to engineering, modelling, communication, entertainment and simulation made us understand that their generative power is not relevant to art and not equivalent to an aesthetic perspective.

\section{AESTHETIC CONSCIOUSNESS}

It goes to the credit of the industry, however, that it recognized the need for aesthetics in tools for practical activity other than art. But while I credit the visionaries-Alan Kay, Ivan Sutherland, Nelson Max, et al.-with this understanding of the formative 
role of the aesthetic component, I must add that it is difficult to say whether the aesthetic component was viewed as a marketing tool, an alibi or an intrinsic part of the design of new tools. Nevertheless, in the databanks of the characteristics of artifacts generated with the aid of computers and aspiring to the status of art, one will find the characteristics commissioned/required by those who supported computer graphics research for flight simulators, radar installations, satellite observation, space exploration, oil exploration, design of new machines (cars and trucks, in particular), etc.

It is comforting to see that money invested in such non-artistic areas trickles down into the hands (and products) of those interested in art. But the inadequacy of such tools for artistic practice remains. The question of precision in art is different from the precision of engineering. The combative nature of art is different from that of the military. An artistic artifact requires a different manufacture than that of mass production. Against the background of the digital (i.e., of the information- and symbol-processing paradigm), we arrive at the realization of the need to consider art in its interrelations with all other products of human activity. The digital computer is the carrier of information and a means of maintaining simultaneous levels of information exchange. It already supports unprecedented forms of human interconnection and makes available new types of interaction. Whatever an artist can do using traditional means will not become more valuable once it is computer generated. It is in the realm of what was not before possible that one can see the assets of this artistic involvement with technology. Digital carriers allow for interactive modes, for integrated environments, for mixed media.

Nevertheless, all this does not come for free. Since more people can participate in making the work, chances are that, in the process, authorship and quite a number of characteristics related to it will change. The digital medium is one of instant replication and perfect fidelity; therefore, the notion of the original, the aura of uniqueness, and the attraction of ownership will have to undergo reinterpretation and change. Our understanding of the artist-public relation changes as the distinction between artist and public gradually disappears. Indeed, in the electronic medium, everything done by an artist can easily be re-processed by the public. Variations become a matter of interaction with the work. The change is from a one-to-many relation to a sequence of one-to-one relations. Even the functions assigned to art change in the sense that an active relation rather than passive contemplation emerges. As I see it, digital art permeates the environment of existence as a neverending process, at levels of quality perhaps far below those celebrated in previous stages of human practice, but reaching far more people (in principle, the entire population). Intensity is converted into extension.

Based on some of these considerations, we should now consider the relation between the possible and the desirable because first, in the age of computer technology, the space of possibilities increases exponentially, and second, in the past, people desired new forms of expression and pushed the technology and the medium of expression to its possible limits. Today, technology leads and actually offers more possibilities than we are able or even qualified to use. Consequently, desirability starts shaping us in our way of expressing convictions, ideals and values. Is it indeed desirable to use a paint program without ever seeing a painting or preparing a canvas, mixing colors, mastering a real brush? Is it acceptable to synthesize sound without knowing what is culturally acknowledged as harmony or tonality? Is it possible to conceive of an electronic sculpture independent of the context of the world for which such a sculpture is produced? I can go on and on with even farther-reaching questions as to the significance of color not only as a component of art, but also with biological implications (its symbolism, its role in memory processes, the effect of the eye's color sensor on each person's well being, the behavioral implications of color in a given culture). Indeed, art is far more than the mere physical presence of an artifact; and this is why the digital approach to art must consider the human being, society and its evolution under new circumstances of life and work [7].

The digital computer made possible an accelerated integration of aesthetic characteristics in non-artistic artifacts. This has contributed to a dissemination of better taste through objects of daily life and in communication. Moreover, the digital computer, together with other electronic and non-electronic technologies, made possible and necessary patterns of human interaction that affect the primacy of language and languageoriented work. We are already entering an age of varied means of expression and communication in which taste, smell and touch as well as images and sounds play ever-increasing roles. Once the dominance of language ceases, we start living in a civilization of several modes of expression and communication. This in turn affects the relation between art and technology as new arts appear and new forms of interaction with art become possible. It is probably worth the effort to understand this diversification as an expression of a new relation between what we call the tools of the artist and the medium.

\section{TOOL OR MEDIUM?}

One question is frequently raised: Is the computer a tool or a medium? The easy answer is: both. But easy answers will not do. In the strictest sense of the word, the computer is neither a tool nor a medium; that is, the programs are the tools, the peripherals (such as printers, plotters, CRTs, sound synthesizers, loudspeakers, etc.) are the medium/media. In creating a sculpture by driving a milling machine, the artist has to understand the relation between the 'virtual' object as it results after data processing and the 'real' artifact that will (or will not) embody desired qualities. Many changes accumulate between the plotted image on the screen and the Cibachrome print; there is a change in quality and quantity between the sound synthesizer and the final tape. This profoundly mediated process, which results in removing the artist from the 'matter' on which he or she acts, requires skills different from those of the traditional craftsperson. It is not that thinking replaces the craft, but it diminishes the importance of craft in the actual making. I feel comfortable with the notion that, in the age of digital technology, the program is the work of art, although I am not quite sure how such a work realizes its meaning. It is probably, because I do not want to discard the thought, through the infinite use of the program, in which case all of us using programs are actually interacting with the art object called 'the program' and thus with the artist as 
author of the program. Whether or not this view is accepted, we still need to make clear that, due to the intrinsic characteristics of digital technology, there is no such thing as a computer artist who is not the author of his or her program. The very few successes we know of are the result of authentic mastery of the programming and the result of the attempt to create a legitimate alternative medium. Harold Cohen created not only the best computational theory of Harold Cohen's art, but also a tool of tremendous flexibility and respectable integrity. For those who want to be Harold Cohen followers, the use of AARON can be rewarding. But whether or not it is Harold Cohen who actually turns on the machine and makes sure it runs, the work is already signed by Harold Cohen; it projects his notion of art, his sensitivity and his particular aesthetic point of view. (AARON does not yet encode Cohen's aesthetics of color, so at least the color component is left to the artist.)

Art is not possible without technology. Nevertheless, art does not reflect how powerful technology is, but how powerfully it serves the artist's artistic means. The need for the 'disappearance' of the technology, for its 'invisibility', has to be put in the perspective of the why of art, as opposed to the how and even the what. In general, when the computer is visible, we are given an indication that the technology is not yet appropriately assimilated in the activity supported. Manfred Mohr and John Pearson are good examples for understanding the implications of this principle. Their work, so different each from the other, is the result of integrating the computer in their thinking about and making of art. Both artists recognize the need for a powerful planning procedure, for an instrument adequate to the research of a personal aesthetic set of possibilities in which geometry plays an important role without becoming a goal in itself. John Pearson confesses that the computer influenced his process of thinking: thus he felt encouraged to look at the many facets of an artistic idea and discovered that some relevant avenues explored in his work would have been overlooked had he relied only on intuition. He typically starts his creative work where the computer ends in generating the shapes that will constitute the invisible support of the final image. Manfred Mohr discovered that his aesthetic interest in multidimensional spaces could not be efficiently supported without an adequate instrument for visualization. He does not continue the tradition of literary descriptions of such higherorder spaces such as Abbot's Flatland [8], but uses a constructive perspective. Curvature, as evidenced in sections of this space, thus transcends the realm of topology and becomes artistically relevant.

Neither Mohr nor Pearson identifies his art as computer generated; and for someone who does not know what goes on in their studios, this is not relevant. At the opposite end of the spectrum are Lillian Schwartz and probably Frieder Nake. There is a strong computer component, almost a declaration of computer identity, that is quite misleading. Actually the computer trademark is a diversion. The expression is not the result of the hardware, but of an analytic effort. Nake's variations on Paul Klee and Lillian Schwartz's Mona Leo (juxtaposing half of the celebrated Mona Lisa and half of Leonardo da Vinci's self-portrait, suggesting that he was the model) are works with a precise aesthetic condition resulting from the integration of the computer in the creative act. The analytic effort does not necessarily become art. Ending with a formal description of a work (like my applications to Brancusi's body of sculpture), the analytic effort constitutes a computational explanation, not a new, original, artistically relevant expression. I give my own work as an example to clarify that the mastery of the computer and the mastery of art are related but still quite different. One does not automatically result from the other.

Can users, the vast majority of those interested in computer art, also succeed? Depending upon what it means to succeed, the answer may be 'Yes'. Provided that we are able to adopt a different notion of art and a different notion of the artist, many arguments speak in favor of an increased interpretive approach, of more performances and larger audiences, and of aesthetic products new in their condition, impact, and cultural and social implications. For all these things to happen, we have to gain access to the technology in each of its various aspects while we simultaneously start-and I mean start-thinking about possibilities, about what is desired, and about what it takes to prepare the creative 'quantum leap' promised by the prog- ress of technology and the experience we are acquiring. My position is that, instead of refusing theory, historical reference, and culture-because some believe that these can obstruct the new and will subtract from our preparedness-we should involve them in our efforts. This becomes so much more critical today since there are very good computational models (i.e., theories) that, while keeping close to the practice, also put this practice in a digital perspective and thus turn out to be instruments of creative understanding [9].

\section{DIVERSIFIED Aesthetic Practice}

At various professional meetings, seminars, workshops and classes during my involvement with computers, I have suggested artistic experiments and new ideas for a creative approach. I have tried some and am still involved in others. Of these, several can be mentioned along the line of the ideas pursued here.

1. Given the integrative power of the technology and the possibilities of combining sound, movement, images, etc., we can create an environment for play that documents itself in the data stored. Eventually a game can be conceived with events taking place both in sequence and in parallel, the outcome being the score for the next game, i.e. participatory performance.

2. Using the networking power of the technology, we can access people in their homes and challenge passivity and complacency by making possible the interaction of all those connected through the cable systems.

3 . We can transform those major events of a democratic society-election debates, congressional debates and votes, referenda, etc.-into major artistic events, not only by recording a vote in a booth, but by making the act of voting an occasion for creative expression.

4. We can make private art part of the community ceremony; we can make possible the display of what people draw, write or compose within a community and allow interactive changes.

5 . We can form our relation to what is already established by 'pumping' into people's homes high-resolution images of museum art; we can allow for interactive programs that will enable the viewer to reframe the work, 
alter it, associate it with other works. These electronic copies will give people a better understanding of both art of the past and new art.

6 . . . more to follow, much more.

Obviously, I am not suggesting that these are the only possible ideas, but that some of them and others, many others, will bring us to a more promising domain than the current use of limited paint programs or sophisticated keyframe electronic animation. But none of these notions is meaningful if we do not build an aesthetic selfconsciousness. Terribly engulfedand justly so-in the technology of computer art, we meet and talk about pixels, megabytes, and call-up color tables. This language is necessary if we want to understand how we do what we do, but not what we do and why we do it. Aesthetic consciousness means the acknowledgment of aesthetic goals and the sharing of aesthetic experience. Frequently, in the absence of such an aesthetic consciousness, we fail to understand our own work. This should make us reflect on our own standards with respect to the work and our discourse about it. The diversity that is possible today will become reality only if we challenge our own prejudices as they have accumulated from prior modes of expression or from recent experiments with technology [10]. It is good that we share programs, that those of us with more resources are willing to disseminate our programs and experience. But this will not lead to more diversity. An animation pipeline used in several universities and abroad will remain uniform unless it is delivered with the firm commitment that it can be altered, that it can and will be creatively redesigned.

And this brings up the final issue: how programs written for particular applications determine the output of so-called artistic attempts. My claim, admittedly expressed in radical language, is that art is made by artists and that a truly creative approach can take place only if we can give the artist an 'empty' computer. What does this mean? Computers are cycles, storage and operating systems, input and out- put devices, compilers and/or interpreters, utilities, procedures. When an artist receives a machine, even with the most basic configuration, the machine already has its pixels defined, its geometry and logic programmed. Whether Boolean logic and art logic are equivalent, reducible to each other, or at least compatible has not been sufficiently researched. But no matter what the answer is, the nature of the machine as predetermined makes it a poor substitute for the empty canvas of the painter, the block of marble of the sculptor, or the blank lined sheets of the composer. We all understand why the computer industry maintains that, for reasons of competition and security, certain limitations (the notion of 'proprietary information') are necessary. But art is 'hacking' and 'viruses', not databank management or increased production. The industry is also preoccupied with providing tools for efficient work, not with the exotic realm of somebody's art. Consequently, the major creative effort of someone really wanting to use this technology for artistic purposes probably involves finding ways to strip the machine and reinvent it in each detail, going into language, interactive modes, and input and output devices. Scientists as well as artists express the same need. "As an algorithm developer, I cannot use a workstation that has specific rendering algorithms already built into it," stated Nelson Max [11]. My own program, as it applies to aesthetic performance, is definitely extreme-an empty computer. But given the background against which it is formulated, chances are that it will be implemented. Small steps in this direction (such as the generalization of associative modes of computation, supported by the hypermedia model) are already noticeable and have encouraged creative applications. Moreover, it may turn out that while the notion of processing is all right, digital formats are not the only type to consider. Binary representation is powerful but, as we know, not necessarily expressive. A compromise between precision and expressive power seems more appropriate and will result not in a computer (the digital machine), but in a family of machines (triadic, tetradic, etc.) that we should be able to interconnect while giving the ana$\log$ a fair chance in the process.

There are many reasons to be optimistic and, although the quality of previous and current work is not among them, I would like to restate my respect for those who have failed. It takes failure, more than success, to open new avenues. In view of the implications of the entire process, it should come as no surprise that a discussion of the emergent aesthetics cannot start with self-delusive value judgments. After all, to discuss aesthetic issues is to discuss the future.

\section{References and Notes}

1. Nicholas Negroponte, "The Return of the Sunday Painter", in The Computer Age Michael L. Dertouzos and Joel Moses, eds. (Cambridge, MA MIT Press, 1979), pp. 21-37.

2. See Mihai Nadin, "The Expansion of Creativ ity vs. Canned Art", keynote address at the lst annual meeting of the Association for Computer Aided Design and Education (ACADE), St. Paul, MN, 18-21 July 1986.

3. Mihai Nadin, "Estetica Generativa (Generative Aesthetics)", in Aesthetics, Information, Algorithm (Bucharest: Ed. Stiintifica, 1971).

4. For more information, see Mihai Nadin "Valoarea Estetica al Algoritmului (Aesthetic Value of the Algorithm)", Tribuna, No. 692, 1968, and "Aleatorism in Modern Art", in Analele universitatii (seria Estetica) 19, No. 1, 105-110 (1970).

5. Mihai Nadin, lecture entitled "Methodological Framework for Computer Graphics and Computer-Aided Design" presented at the conference "The Designer and the Technological Revolution" at the Rochester Institute of Technology, 13-15 May 1982

6. Paul S. Heckert, "Ray Tracing Jell-O Brand Gelatin: New Technology Is Presented for Imaging a Restricted Class of Dessert Foods", Com munications of the ACM 31, No. 2, 131-134 (February 1988).

7. Mihai Nadin, "Image Machine and Artificial Inte!ligence", keynote address at "Ars Electronica", Linz, Austria, 16-21 September 1987.

8. Edwin Abbot, Flatland: A Romance of Many Dimensions (New York: New American Library, 1984)

9. For an in-depth analysis, see Mihai Nadin, "The Meaning of the Visual: 12 Theses Regarding the Visual and Its Interpretation", Semiotica 52 No. 3/4 (1984).

10. Refer to Mihai Nadin, "Values in Post-Modern Art", in Semiotics 1983 (Lanham/New York/ London: University Press of America, 1987).

11. Karen A. Frankel, "The Art and Science of Visualizing Data", Communications of the ACM 31, No. 2, 111-122 (February 1988) 